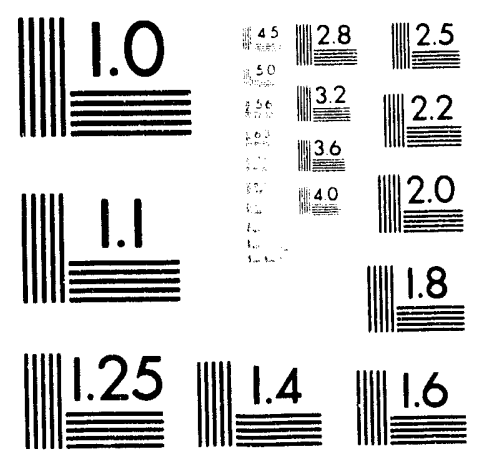



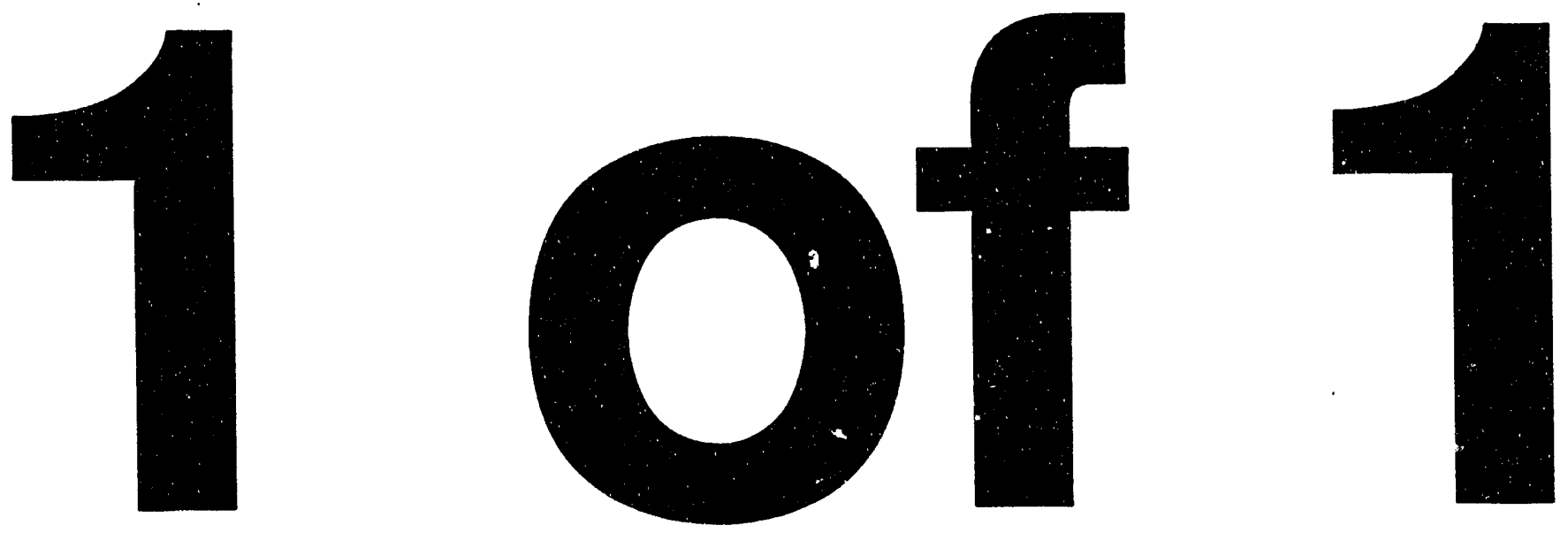


\section{CONDUCTORS WTH SMALL FERMI ENERGIES AND SMALL GAP ENERGIES.}

R. J. Thorn

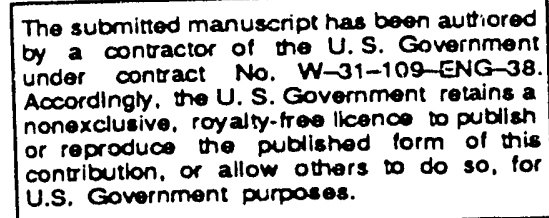

\section{DISCLAIMER}

This report was prepared as an account of work sponsored by an agency of the United States Government. Neither the United States Government nor any agency thereof, nor any of their employees, makes any warranty, expiess or implied, or assumes any legal liability or responsibility for the accuracy, completeness, or usefulness of any information, apparatus, product, or process disclosed, or represents that its use would not infringe privately owned rights. Reference herein to any specific commercial product, process, or service by tr.de name, trademark, manufacturer, or otherwise does not necessarily constitute or imply its endorsement, recommendation, or favoring by the United States Government or any agency thereof. The views and opinions of authors expressed herein do not necessarily state or reflect those of the United States Government or any agency thereof.

$$
\begin{array}{r}
f \ldots g s \\
\cdots \& g
\end{array}
$$




\title{
CONDUCTORS WITH SMALL FERMI ENERGIES AND SMALL GAP ENERGIES.
}

\author{
R. J. Thorn \\ Materials Science and Chemistry Divisions \\ Argonne National Laboratory \\ Argonne, IL 60439
}

\section{Abstract}

If the Fermi energy is of the order of meV's, the usual treatment uf the density of free electrons is not valid, but use can be made of an averaged density of states that depends weakly on temperature, so that the temperature variation of the conductivity can be expressed by the equation:

$$
\left.\sigma \cong \mathrm{CT}^{(1-\mathrm{s})} \ln \left\{\left[\left(\exp \left(\beta \mathrm{E}_{\mathrm{f}}\right)+1\right) / 2\right]\left[\exp \left(-\beta\left(\mathrm{E}_{\mathrm{g}}-\mathrm{E}_{\mathrm{f}}\right)\right)+1\right)\right]\right\}
$$

in which $E_{f}$ is the Fermi energy, $E_{g}$ is the top of the energy gap for thermal activation, $s$ is the exponent of the temperature-dependent scattering. This equation serves to define a class of solids consisting of a microcomposite with a narrow conduction band for which $\mathrm{E}_{\mathrm{f}}$ of the order of ceV's or less and a thermial activated conduction for which $E_{g}$ is of the order of ceV's. It describes quantitatively the conductivity, $\sigma(\mathrm{T} ; \delta)$, for $\mathrm{YBa}_{2} \mathrm{Cu}_{3} \mathrm{O}_{7}-\delta$ and $\sigma(\mathrm{T} ; \mu)$ as the hydrostatic pressure, $\mathrm{p}$, is varied for $\kappa^{-}$ $(\mathrm{BEDT}-\mathrm{TTF})_{2} \mathrm{CuN}(\mathrm{CN})_{2} \mathrm{Br}$.

\section{Fermi Energies of the Order of eV's.}

In the usual treatment ${ }^{1}$ of the free electron in solids, use is made of the fact that for a sufficiently large Fermi energy the derivative of the Fermi-Dirac distribution function with respect to energy,

$$
-\partial \mathrm{f}(\varepsilon ; \mathrm{T}) / \partial \varepsilon=\beta\left[\exp \beta\left(\varepsilon-\mathrm{E}_{\mathrm{f}}\right)\right]\left\{\exp \left[\beta\left(\varepsilon-\mathrm{E}_{\mathrm{f}}\right)\right]+1\right\}^{-2},
$$

is a narrowly peaked function for the temperatures usually employed in the measurements (temperatures of the order of $1000 \mathrm{~K}$ ) so th.at $(\varepsilon-$ $\left.\mathrm{E}_{\mathrm{f}}\right) / \mathrm{kT}>1$. Then one can employ the integration by parts,

$$
\int[\mathrm{dF}(\varepsilon) / \mathrm{d} \varepsilon] \mathrm{f}(\varepsilon ; \mathrm{T}) \mathrm{d} \varepsilon=-\int \mathrm{F}(\varepsilon)[\partial \mathrm{f}(\varepsilon ; \mathrm{T}) / \partial \varepsilon] \mathrm{d} \varepsilon,
$$

and express $F(\varepsilon)$ as a Taylor series in $\left(\varepsilon-E_{f}\right) / k T$ to evaluate the integral 


\section{2}

on the left side of equation (2). This means that only electrons with energies near the Fermi energy make a significant contribution.

This situation is generally used in discussions of the conductivities of metals and is intended to apply to the normal stace of the superconducting oxides and the organic charge transfer conductors.

\section{Fermi Energies of the Order of meV's.}

One of the most significent but overlooked features of the oxide and organic conductors is the fact that the conductivities are measured at and below $300 \mathrm{~K}$. At these temperatures, $\mathrm{kT}=2.6 \times 10^{-2} \mathrm{eV}$ or less and the states having energies somewhat larger than this value are essentially bound, so that they do not contribute significantly. Wherever semiconductivity is observed with these materials, the thermal activation energy is small, i.e., of the order of $0.05 \mathrm{eV}$ or smaller. Because the change from semiconductive to metal-like behavior, usually through changes in composition or hydrostatic pressure $\mathbf{2 - 8}$ is apparently continuous, it is doubtful that any Fermi enr.rgy associated with the metal-like variation is any larger than the $2.6 \times 10^{-2} \mathrm{eV}$. At the very least, one must examine the situat on wherein $E_{\mathrm{f}}$ is much smaller than that required in the usual expansion associated with equation (2).

A comparison of -- $\partial \mathrm{f}(\varepsilon ; \mathrm{T}) / \partial \varepsilon$ vs $\varepsilon$ when $\mathrm{E}_{\mathrm{f}}$ is $0.1 \mathrm{eV}$ or iess (Figure 1 , curves $b-d$ ) with this function when $E_{f}$ is $1 \mathrm{eV}$ or larger (Figure 1, curve a) reveals a difference that has been little recognized apparently heretofore. With $-\partial \mathrm{f}(\varepsilon ; \mathrm{T}) / \partial \varepsilon$ vs $\varepsilon$ shown in Figure 1 , one cannot always state that only electrons with energies near the Fermi energy contribute, because the function is not always peaked. The variation for $E_{\mathrm{f}}=10^{-3}$ $\mathrm{eV}$ is such that $-\partial \mathrm{f}(\varepsilon ; \mathrm{T}) / \partial \varepsilon$ increases with decreasing energy starting near the Fermi energy and then attains a nearly constant value as $\varepsilon$ approaches zero (See Figure 1)). This shape does not depend on temperature

The variations of the Fermi Dirac distribution function, $\mathrm{f}(\varepsilon ; \mathrm{T})$ vs $\varepsilon$, at $300 \mathrm{~K}$ and for $\mathrm{E}_{\mathrm{f}}$ at $1,0.1,0.05$ and $0.001 \mathrm{eV}$ are shown in Figure 2 . When the Fermi energy is of the order of eV's, as is the case in the discussions of metals, the transition from nearly full occupancy to nearly empty occurs over a small range in energies, whereas when the Fermi energy is of the order of meV's the occupation increases slowly as $\varepsilon$ is decreased and approaches a value significantly less than full occupancy as the energy approaches zero.zero.

\section{Semimetal with Small Fermi Energy.}


3

To evaluate the integral,

$$
\int \mathrm{g}(\varepsilon) \mathrm{f}(\varepsilon ; \mathrm{T}) \mathrm{d} \varepsilon
$$

for $0 \leqq \varepsilon \leqq E_{\mathrm{f}}$ with small Fermi energies, one can make use of the averaged value of $g(\varepsilon)$ defined by

$$
<g\left(E_{\mathrm{f}}, \mathrm{T}\right)>=\left\{\int_{0}^{\varepsilon f} \mathrm{~g}(\varepsilon) \mathrm{f}(\varepsilon ; \mathrm{T}) \mathrm{d} \varepsilon\right\} /\left\{\int_{0}^{\varepsilon f} \mathrm{f}(\varepsilon ; \mathrm{T}) \mathrm{d} \varepsilon\right\}
$$

provided that the dependence of the averaged value on teinperature is known or is negligible (See Addenda).

If $g(\varepsilon)$ is expanded in a Taylor series in $\left(\varepsilon-E_{f}\right)$, one finds that with $\left(\varepsilon-E_{f}\right)<1$ :

$$
\begin{aligned}
\int_{0}^{E r} \mathrm{~g}(\varepsilon) \mathrm{f}(\varepsilon ; \mathrm{T}) \mathrm{d} \varepsilon= & \sum_{n \geqslant 0}\left[\mathrm{~g}^{\mathrm{n}}\left(\mathrm{E}_{\mathrm{f}}\right) / \mathrm{n} !\right] \int_{0}^{E f}\left(\varepsilon-\mathrm{E}_{\mathrm{f}}\right)^{\mathrm{n}} \mathrm{f}(\varepsilon ; \mathrm{T}) \mathrm{d} \varepsilon \\
& =\left\langle\mathrm{g}\left(\mathrm{E}_{\mathrm{f}}, \mathrm{T}\right)>\int_{0}^{\mathrm{eg}} \mathrm{f}(\varepsilon ; \mathrm{T}) \mathrm{d} \varepsilon\right.
\end{aligned}
$$

$\mathrm{g}^{\mathrm{n}}\left(\mathrm{E}_{\mathrm{f}}\right)$ is the $\mathrm{nth}$ derivative of $\mathrm{g}(\varepsilon)$ evaluated at $E_{\mathrm{f}}$.

Thus, this procedure has separated the intergal with the integrand, $g(\varepsilon) f(\varepsilon, T)$, into two factors, one of which depends on the density of states at the Fermi energy and weakly on temperature (see Addenda) and the other of which depends only on the Femi-Dirac distribution.

For the density of carriers with energies of $\mathrm{C} \leq \varepsilon \leq \mathrm{E}_{\mathrm{f}}$, one can now write if $E_{\mathrm{f}}$ is sufficiently small:

$$
\begin{gathered}
\mathrm{N}\left(\mathrm{T} ; \mathrm{E}_{\mathrm{f}}\right)=\int_{0}^{\mathrm{Ef}} \mathrm{g}(\varepsilon) \mathrm{f}(\varepsilon ; \mathrm{T}) \mathrm{d} \varepsilon=\mathrm{g}\left(\mathrm{E}_{\mathrm{f}}\right) \beta^{-1} \ln \left\{\left[\exp \left(\beta \mathrm{E}_{\mathrm{f}}\right)+1\right] / 2\right\}+ \\
\sum_{n \geq 1}\left[\mathrm{~g}^{\mathrm{n}}\left(\mathrm{E}_{\mathrm{f}}\right) / \mathrm{n} !\right] \int_{0}^{\mathrm{Er}}\left(\varepsilon-\mathrm{E}_{\mathrm{f}}\right)^{\mathrm{n}} \mathrm{f}(\varepsilon ; \mathrm{T}) \mathrm{d} \varepsilon .
\end{gathered}
$$




\section{4}

For $E_{\mathrm{f}}=0.001 \mathrm{eV}$ and $\mathrm{T}=200 \mathrm{~K}$, the first term on the right side of equation (7) is $5 \times 10^{-4} \mathrm{~g}\left(\mathrm{E}_{\mathrm{f}}\right)$ (zeroth term in the total summation) and the first term in. the summation shown is $-2 \times 10^{-7} \mathrm{~g}^{\prime}\left(\mathrm{E}_{\mathrm{f}}\right)$

\section{The Total Density of Carriers.}

The expression for $N(T)$ requires that the integral over the integrand, $\mathrm{g}(\varepsilon) \mathrm{f}(\varepsilon ; \mathrm{T})$, be evaluated in general over the limits $0 \leq \varepsilon \leq \infty$, i.e., over the conduction and valence bands, at least. Hence one writes:

$N(T)=\int_{0}^{\infty} g(\varepsilon) f(\varepsilon ; T) d \varepsilon=\int_{0}^{E f} g(\varepsilon) f(\varepsilon: T) d \varepsilon+\int_{E g}^{\infty} g(\varepsilon) f(\varepsilon ; T) d \varepsilon$

The interval $E_{\mathrm{f}} \leq \varepsilon \leq \mathrm{E}_{\mathrm{g}}$ can be omitted, because generally there is a gap between the conduction and valence bands.

The $g(\varepsilon)$ in the integand of the second intergal on the right side of equation (8) can be expanded in series in $\left(\varepsilon-E_{g}\right)$, and use can be make of the expression:

$$
g\left(E_{g}\right)=g\left(E_{f}\right)+\sum_{n \geq 1}\left(E_{g}-E_{f}\right)^{n} g^{n}\left(E_{f}\right) / n !
$$

One then finds for the second integral:

$$
\begin{gathered}
\mathrm{N}\left(\mathrm{T} ; \mathrm{E}_{\mathrm{g}}\right)=\mathrm{g}\left(\mathrm{E}_{\mathrm{f}}\right) \beta^{-1} \ln \left\{\exp \left[\beta\left(\mathrm{E}_{\mathrm{g}}-\mathrm{E}_{\mathrm{f}}\right)\right]+1\right\}+ \\
\sum_{n \geq 1}\left[\left(\mathrm{E}_{\mathrm{g}}-\mathrm{E}_{\mathrm{f}}\right)^{\mathrm{n}} \mathrm{g}^{\mathrm{n}}\left(\mathrm{E}_{\mathrm{f}}\right) / \mathrm{n} !\right] \int_{\mathrm{Eg}_{\mathrm{g}}}^{\infty} \mathrm{f}(\varepsilon ; \mathrm{T}) \mathrm{d} \varepsilon+ \\
\sum_{n \geq 1}[\mathrm{~g}(\varepsilon) / \mathrm{n} !] \int_{\mathrm{E}_{\mathrm{g}}}^{\infty}\left(\varepsilon-\mathrm{E}_{\mathrm{g}}\right)^{\mathrm{n}} \mathrm{f}(\varepsilon ; \mathrm{T}) \mathrm{d} \varepsilon .
\end{gathered}
$$

For $\mathrm{E}_{\mathrm{f}}=0.001 \mathrm{eV}, \mathrm{Eg}=0.01$, and $\mathrm{T}=200 \mathrm{~K}$, one finds:

$$
\mathrm{g}\left(\mathrm{E}_{\mathrm{f}}\right) \beta^{-1} \ln \left\{\exp \left[\beta\left(\mathrm{E}_{\mathrm{g}}-\mathrm{E}_{\mathrm{f}}\right)\right]+1\right\}=8 \times 10^{-3} \mathrm{~g}\left(\mathrm{E}_{\mathrm{f}}\right)
$$

the first term in the first summation $=7 \times 10^{-5} \mathrm{~g}^{\prime}\left(\mathrm{E}_{\mathrm{f}}\right)$,

and the first term in the second summation $=2 \times 10^{-4 . .} g^{\prime}\left(E_{g}\right)$.

If $g(\varepsilon)$ is a slowly varying function such that $g^{\prime}\left(E_{f}\right)$ and $g^{\prime}\left(E_{g}\right)$ are less 
5

that the order of $g\left(E_{\mathrm{f}}\right)$, then one can set aside the summation terms in equations (7) and (10) and use the zeroth order forms. At present sufficient information is not available concerning the density of states. The values calculated from band theory are not applicable for two reasons: (a) In these calculations, it is usually assumed that the density of carriers is known to be of the order of $10^{22} \mathrm{~cm}^{-3}$, whereas the small Fermi energies involved in this study implies a value smaller by a few orders of magnitude and (b) as will be shown subsequently, the materials of interest conduct by small polarons, so that the correct calculation must be based on electron-phonon coupling. In any event the structure of the conductivity vs temperature with the zeroth approximation is used to study the possible significence of these equations.

\section{V.Conductivity.}

If it is possible to define some averaged scattering relaxation time in the phenomenological expression for the conductivity, then one can write

$$
\sigma(\mathrm{T})=\mathrm{N}(\mathrm{T}) \mathrm{e}^{2}<\tau(\mathrm{T})>/ \mathrm{m} .
$$

For the variation of the scattering time with temperature, one can write that $\langle\tau(T)\rangle \propto T^{-s}$ with $s=1$ for phonon-electron scattering and $s=2$ for electron-electron scattering. The equation for the conductivity can then be written:

$$
\left.\sigma\left(T ; E_{f}, E_{g}, s\right) \cong C T^{(1-s)} \ln \left\{\left[\exp \left(\beta E_{f}\right)+1\right] / 2\right]\left[\exp \left[-\beta\left(E_{g}-E_{f}\right)\right]+1\right]\right\}
$$

A study of this equation shows that the structure is attributable mostly to the variation of $E_{f}$, i.e., the chemical potential of the electrons wherever they are located, at constant $E_{g}, s$, and $\left\langle g\left(E_{f}\right)>\right.$. Such is shown in Figure 3 with $\mathrm{E}_{\mathrm{g}}=2.5 \times 10^{-2} \mathrm{eV}, \mathrm{s}=2$, and $\mathrm{O} \leq \mathrm{E}_{\mathrm{f}} \leq 2.5 \times 10^{-2} \mathrm{eV}$. Curve f illustrates the fact that within a high precision the linear variation of $\rho(\mathrm{T})$ assumed to be characteristic of the superconducting composition is also included in the equation 9

This equation serves to define a class of materials characterized by Fermi energies of the order of ceV's or less and by thermal activation energies of the order of ceV's, by densities of state that vary slowly with energy, and in which the systematic variation of $\sigma$ (T;parameter) is determined mostly by the chemical potential of the electrons wherever they are located.

The $\sigma(T)^{\prime}$ 's for several of the superconducting oxides 10 and of $\kappa$ $(\mathrm{BEDT}-\mathrm{TTF})_{2} \mathrm{Cu}(\mathrm{NCS})_{2} \mathbf{1 1}$ are described quantitatively by equation (15). 
The only other equation that I have found to have this structure is that first published by Holstein $\mathbf{1 2}$ for a model of a small polaron in a one dimensional molecular crystal. His equation describes thermal hopping that dominates at the higher temperatures and narrow band.tunneling that dominates at the lower temperatures The structure of his equaion is the same as that shown in rigure 6 . His estimate of the width of the narrow band is consistent with the Fermi energies derived from measurements of $\sigma(\mathrm{T})$ cited below.

Evidence of the significance of equation (15) and Holstein's equation is contained in the variation of the conductivity with temperature at various hydrostatic pressures for $\kappa-(\mathrm{BEDT}-\mathrm{TTF})_{2} \mathrm{CuN}(\mathrm{CN})_{2} \mathrm{Br}$ reported by Sushko et al $^{3}$. These measurements were made on a single crystal that showed no evidence of superconductivity at temperatures near the maxima in the $\rho(T ; \mathrm{p})$ 's; the transition to the superconducting state occurred near $10 \mathrm{~K}$. The curves obtained by Sushko et al are described quantitatively $\mathbf{8}$ by both equation(15) and Holstein's equation.

The evidence given by the $\rho(\mathrm{T} ; \delta)$ 's for $\mathrm{YBa}_{2} \mathrm{Cu}_{3} \mathrm{O}_{7}-\delta$ is not quite so definitive, because the rapid decreases in $\rho(\mathrm{T})$ 's on the low temperature side of the maxima can be obscured by the onset of the superconducting state. However for a curve like b in Figure 4, the diamagnetic susceptibility is so extremely small that its contribution is probably insignificant 13. Hence, it is plausible to accept for the present at least these equations as quantitative descriptions of the normal state of the superconducting oxides and the organic charge transfer compounds and to examine what this means with respect to the onset of the superconducting state. A bipolaronic state is certainly indicated.

The narrow-band tunneling is one of the "striking" features of the small polaron theory 14. However, experimental evidence for it has been so lacking heretofore that some have doubted that it could ever be observed. Besides the evidence presented herein, observations by Kadono et al $\mathbf{1 5}$ may also reveal this phenomenon. In a study of the low temperature diffusion of positive muons in copper, they found that the hopping rate decreases between $135 \mathrm{~K}$ and approximately $30 \mathrm{~K}$ andit increases slowly between $30 \mathrm{~K}$ and approximately $0.15 \mathrm{~K}$. Above $100 \mathrm{~K}$, the behavior is consistent with the Arrhenius type variation, but below approximately 15 $\mathrm{K}$ the behavior appears to be described by "band-like motion of light interstitials", an effect similar to the narrow-band tunneling in the small polaron theory 16 .

It has been approximately 33 years since Holstein published tra 
7

equations that predict this class of materials, a class in which apparently the existence of the two mechanisms in the normal state is a requirement for the onset of the superconducting state. It is especially intriguing to imagine the significance of the coritribution of the tunneling mechanism to the superconducting state. The recognition of all this is indeed a tribute to the work of Holstein.

\section{Addenda.}

1. Temperature Dependence of $\left\langle\mathrm{g}\left(\mathrm{E}_{\mathrm{f}} ; \mathrm{T}\right)\right\rangle$

If one examines each term of the derivative in the expansion of $g\left(E_{f}, T\right)$ and each corresponding term of $g\left(E_{\mathrm{f}} \cdot \mathrm{T}\right)$, one can write that

$$
\left(\partial<\mathrm{g}\left(\mathrm{E}_{\mathrm{f}}, \mathrm{T}\right)>/ \partial \Upsilon\right)_{\mathrm{n}} /\left(<\mathrm{g}\left(\mathrm{E}_{\mathrm{f}} ; \mathrm{T}\right)>\right)_{\mathrm{n}}=\left\{\mathrm{F}^{\mathrm{o}} \mathrm{F}^{\mathrm{n}}-\mathrm{F}^{\mathrm{n}} \mathrm{F}^{\prime} \mathrm{o}\right\} \mathrm{F}^{\mathrm{o} / \mathrm{F}^{\mathrm{n}}}
$$

In this equation

$$
\begin{aligned}
& F^{n}=\int_{0}^{\varepsilon f}\left(\varepsilon-E_{f}\right)^{n} f(\varepsilon ; T) d \varepsilon \\
& F^{n}=\int_{0}^{E r}\left(\varepsilon-E_{f}\right)^{n}(\partial f(\varepsilon ; T) / \partial T) d \varepsilon .
\end{aligned}
$$

The first term in equation (16) is zero. The second term is of the order of $10^{-14}$, so that the convergence appears to be sufficiently rapid that one can neglect the temperature dependence of $\langle\mathrm{g}(\varepsilon ; \mathrm{T})\rangle$.

\section{The Small Fermi Energy}

In relation to the usually accepted values for the Fermi energy of the superconducting oxides and the organic charge transfer compounds, the small values cited above appear to be unexpected However, the logic used to derive the small values is sufficiently definitive for the Fermi energy and sufficiently quantitative that the conclusion cannot be dismissed on the basis that the small values do not agree with the usually assumed values for the density of carriers. Besides, there are other studies that indicate that the unusual properties of these materials can be understood in terms of small Fermi energies. Kresin and Wolf $\mathbf{1 7}$ have calculated the Fermi energy for $\mathrm{YBa}_{2} \mathrm{Cu}_{3} \mathrm{O}_{7}-\delta$ with $\delta$ near 0.1 from measured values of the heat capacity and an estimated value for the density of carriers:

$$
E_{f}=\left(\pi^{2} k^{2} N\right) /(6 \gamma)
$$




\section{8}

in which $\gamma$ is the proportionality constant between heat capacity and temperature. They estimated $\mathrm{N}$ at $5 \times 10^{21} \mathrm{~cm}^{-3}$ and obtained $\mathrm{E}_{\mathrm{f}}=0.07$ $\mathrm{eV}$. If $\mathrm{N}$ is estimated at $3.5 \times 10^{20} \mathrm{~cm}^{-3}$, then, $\mathrm{E}_{\mathrm{f}}=5 \times 10^{-3} \mathrm{eV}$.

In a series of articles by Ashkenazi and colleagues $\mathbf{1 8}$, the measured values for the thermal electric power over temperatures ranging from 100 to $1200 \mathrm{~K}$ have been initerpreted in terms of a narrow band model similar to that for a small polaron. In this model the band width is considered to be narrow "when it is comparable or even the same order of magnitude as $\mathrm{kT}$." For $\mathrm{T}=100 \mathrm{~K}, \mathrm{kT}=8.6 \times 10^{-3} \mathrm{eV}$.

\section{Small Polarons in Mixed Valent Systems.}

The role of mixed valency in the transfer of charge has been a subject of numerous studies in chemistry ${ }^{\mathbf{1 9}}$, and the theory of such has been published in the chemical literature 20. Small polarons, on the otherhand, have been the subject studied primarily by physicists, and starting with Holstein's articles, the theory has been developed in the physic's literature Recently, primarily with the advent of the superconducting oxides, it has been recognized that the two phenomena are interrelated $\mathbf{1 3 , 2 1 , 2 2}$. An examination of the theory presented by Piepho, Krauze, and Schatz 20 reveals that the basis is essentially the same as that of Holstein.

In the case of $\mathrm{YBa}_{2} \mathrm{Cu}_{3} \mathrm{O}_{7}-\delta$, sufficient experimental evidence exists to enable one to make a reasonably reliable description that is consistent with the indication that the current is carried by small polarons. From $X$-ray photoelectron spectroscopic measurements by Steiner et al $\mathbf{2 3}$ and the neutron diffraction structural investigations of Jorgensen et al ${ }^{\mathbf{2 4}}$, one can deduce $\mathbf{2 1}$ that the configurational equilibria,

$$
(\mathrm{Cucs})^{3+}(\mathrm{Oas})^{2-}=(\mathrm{Cucs})^{2+}(\mathrm{Oas})^{1-}
$$

and

$$
(\mathrm{Oas})^{2-} \text { as }=(\mathrm{Oas})^{1-\mathrm{as}^{1-}}
$$

exist in this oxide. Thus at the microscopic level, the oxygen ions at the $\mathrm{O} 1$ positions in the basal piane of $\mathrm{YBa}_{2} \mathrm{Cu}_{3} \mathrm{O}_{7}-\delta$ vibrate between the copper $\mathrm{Cu} 1$ positions and with some frequency transfer charge. Hence, an adequate density of states must be vibronic.

Although it may have been known that the organic charge transfer conductors involve mixed valency, the extent and significance of such has not been in general appreciated. Kurmoo and Kanazawa 25 recognized that mixed valency occurs in the organic moiety, the 
9

inorganic moiety, or both. However, they did not recognize that it is the potential for the mixed valency that is the significant feature. Even though the charge on the organic radical cation may appear be integral, complete charge transfer certainly does not occur and the sulfur must be considered to be potentially in a mixed valent state.

Thus the organic charge transfer compounds generally have mixed valent species in both the cation and the anion. In the (BEDT-TTF $)_{2}{ }^{1+}$ the sulfur can exist potentially in at least three states: $\mathrm{S}^{1-}, \mathrm{S}^{0}, \mathrm{~S}^{1+}$; in the anion [CuN(CN)Br] ${ }^{1-} \mathrm{Cu}, \mathrm{N}$ and $\mathrm{Br}$ can be dual valent, so that configurational equilibria comparible to (20) and (21) exist in these materials 8 .

Some confusion may exists concerning the conceptualization of the small polaron. In some cases, it is imagined to consist of a self-trapped, phonon-coupled electron in a locally distorted lattice of an ionic solid and is considered to be relatively immoble $\mathbf{2 6}$. The small polaron imagined herein is more like the small polaron imagined by Holstein in a molecular crystal and the one imagined by Böttger and Bryksin 27. The two concepts may appear to be quite different, but perhaps the difference is one of degree rather than kind. In $\mathrm{YBa}_{2} \mathrm{Cu}_{3} \mathrm{O}_{7}-\delta$ with $\delta$ near 0.1 , most of the configurations in the basal plane are $(\mathrm{Cucs})^{3+}(\mathrm{Oas})^{2-}$, but there is a small concentration of $(\mathrm{Cucs})^{2+}(\mathrm{Oas})^{1+}$ that distorts the matrix lattice and can accordingly be imagined to be similar to the self-trapped electron. However in the ionic crystal like NaF, the energies of the configurational equilibria equivalent to those illustrated in equations (20) and (21) are so large that the small polaron in this solid is relatively immoble.

\section{Acknowledgement}

I thank J. M. Williams for the opportunity to accomplish this research. I thank A. M. Stoneham for bring to my attention the measurements with the positive muons and J. Ashkenazi for the evidence for the small Fermi energy given by the measurements of the thermoelectric power. Work at Argonne National Laboratory is sponsored by the U.S Department of Energy, Office of Basic Energy Sciences, Division of Materials and Chemical Sciences, under contract W-31-109 ENG-38. 


\section{0}

\section{References}

1. F. Seitz, Modern Theory of Solids, McGraw Hill Book Co., Inc., N.Y. 1940, pp.147-150; F. J. Blatt, Physics of Electronic Conduction in Solids., McGraw Hill Book Co, N.Y., 1968, pp. 59-62.

2. A. M. Kini, U. Geiser, H-C. Kato, K. D. Carlson, H. H. Wang, M. R. Montaghan, J. M. Williams, Inorg. Chem., 26 (1897) 1834. .

3. Yu. V. Sushko, V. A. Bondarenko, R. A. Petrosov, N. D. Kushch, and E. B. Yagubskii, J. Phys. France I, 1 (1991) 1375.

4 Yu Mei, C. Jiang, S. M. Green, H. L. Luo, and C. Politis, Z. Phys. B Condensed Matter, $\underline{69}$ (1987) 11.

5 H. Takagi, S. Uchida, H. Iwabuchi, H. Eisaki, K. Kishio, K. Kitazawa, K. Fueki, and S. Tanaka, Physica B 148 (1987) 349.

6. H. Mazaki, Y Ueda, Y. Aikara, T. Kubozoe, and K. Kosuge, Jpn. J. Appl. Phys., 28 (1989) L368

7 K. Suenago and G. Oom Appl Phys., 28 (1989) L368.i, J. Phys. Soc. Jpn., 60 (1991) 1189.

8. R. J. Thorn, preprint, Role of Hydrostatic Pressure in the Conductivity of $\kappa-(B E D T-T T F)_{2} C u N(C N)_{2} B r$, June 1992.

9. R. J. Thorn, preprint, Variation of Conductivity with Temperature for the Normal State of Oxide Superconductors, July 1992.

10. R. J. Thorn and C. E. Thorn, J. Phys. Chem. Solids, 50 (1989) 153.

11. C. S. Cariss, L. C. Porter, and R. J. Thorn, Solid State Commun. 74 (1990) 1269.

12. T. Holstein, Ann Phys., $\underline{8}$ (9959) 325,343

13. R. J. Thorn, Physica C, 190 (1992) 193.

14. I. G. Austin and N. F. Mott, Adv. Phys., 18 (1969) 41.

15. R. Kadono, J. Imazato, K. Nishiyama, K. Nagamine, T. Yamazaki, D. Richter, and J. M. Welter, Phys. Lett., 109A (1985) 61.

16. S. F. J. Cox and A. M. Stoneham, Muon Beams, Used for Studying the Solid State, Science and Engeering Research Council, Rutherford, . Laboratory, RAL-92-002, Didcot, U. , January 1992.

17. V. Z. Kresin and S. A. Wolf, Novel Superconductivity (Editors, S. A. Wolf and V. Z. Kresin) Plenum Press, N. Y., 1987, pp 287-291;

V. Z. Kresin and S. A. Wolf, Solid State Commun.,63 (1987) 1141; V. Z. Kresin, G. Deutscher, and S. A. Wolf, High Temperature Superconductivity (Editors, R. Nicolsky, R. A. Barrio, O. Ferreiro de Lima, and R. Escudero) World Scientific, N. J., 1989, pp. 159-165.

18. J. Genossar, B. Fisher, I. O. Lelong, Y. Ashkenazi, and I. Patlagan, Physica C, 157 (1989) 320; B. Fisher, J. G. Enossar, L. Patlagan, I. O. Lelong, and J. Ashkenazi, Physica C, 162-164 (1989) 1207; J. Genossar, B. Fisher, and J. Ashkenazi, Physica C, 162-164 (1989) 1015; B. Fisher, J. Genossar, I. O. Lelong, A. Kessel, and J. Ashkenaz1, J. Superconductivity, 1 (1988) 53; J. Ashkenazi, B. 
Fisher, J. Genossar, A. Kessel, and I. O. Lelong, Helv. Phys. Acta., $\underline{61}$ (1988) 433.

19. Mixed Valency Systems: Applications in Chemistry, Physics, and Biology (Edited by K. Prassides) Kliwer Academic Publishers, Dordrecht, Netherlands, 1991.

20. See S. B. Pieho, E. R. Krausz, and P. N. Schatz, J. Am. Chem. Soc., 100 !978) 2996.

21. R. J. Thorn, The Physics and Materials Science of Superconductivity II (Edited by R. Kossowsky, B. Reveau, D. Wollenbend, and S. K. Patapis) Kluwer Academic Publishers, Dordrech, Netherlands, 1992, pp. 255-282.

22. L. J. deJongh, Physica C, 152 (1988) 171; L. J. deJongh, Mixed Valency Systems: Applications in Chemistry, Physics, and Biology (Edited by K. Prassides) p. 223,Kluwer Academic Publishers, 1991.

23 P. Sieiner V. Kinsinger, I. Sander, B. Siegwart, S. Hùfner, and C. Politis, Z. Phys., B, Condensed Matter, 67 (1987) 19.

24 J. D. Jorgensen, M. A. Beno, D. G. Hinks, L. Soderholm, K. Violin, K J. Hitterman, J. D. Crace, I. K. Schuller, C. V. Segre, K. Zhang, and M.S. Kleesfisch, Phys. Rev., B 36 (1987) 3608.

25 M. Kurmoo, D. Kanazawa, and P. Day, Mixed Valency Systems: Applications in Chemistry, Physics, and Biology (Edited by K. Prassides) p. 419, Kluwer Academic Publishers, Dordrecht, Netherlands, 1991 . .

26. D. Emin, private communication.

27. H. Böttger and V. V. Bryksin, Phys. Stat. Sol., (b) 67 1975) 583; Hopping Conduction on Solids, VCH Publishers, Deerfield Beach, FL, 1895. 


\section{2}

\section{Figure Captions}

Figure 1. Plot of derivative of Fermi Dirac distribution function with respect to energy, $-\partial \mathrm{f}(\varepsilon, \mathrm{T}) / \partial \varepsilon$ vs $\log \varepsilon$, at $300 \mathrm{~K}$ for Fermi energies: (a) $E_{f}=1 e V$, (b) $E_{f}=0.1 \mathrm{eV}$, (c) $E_{f}=0.05 e V$, and (d) $E_{f}$ $=0.001 \mathrm{eV}$.

Figure 2. Plot of Fermi Dirac distribution function, $\mathrm{f}(\varepsilon, \mathrm{T})$ vs $\log \varepsilon$, at $300 \mathrm{~K}$ for Fermi energies: (a) $E_{\mathrm{f}}=1 \mathrm{eV}$, (b) $E_{\mathrm{f}}=0.1 \mathrm{eV}$, (c) $E_{\mathrm{f}}=0.05$ $\mathrm{eV}$, and (d) $E_{\mathrm{f}}=$ (d) $E_{\mathrm{f}}=0.001 \mathrm{eV}$.

Figure 3. Plots of normalized resistivities vs temperature calculated with equation (15) with a constant gap-energy $\left(2.5 \times 10^{-2} \mathrm{eV}\right)$, a constant scattering exponent $(\mathrm{n}=2)$, and varying small Fermi energies: (a) $E_{\mathrm{f}}=0$, (b) $2 \times 10^{-4}$, (c) $3.25 \times 10^{-4}$, (d) $5 \times 10^{-4}$, (e) $2 \times 10^{-3}$, and (g) $2.5 \times 10^{-2} \mathrm{eV}$ ). Curve $\mathrm{f}$ is derived from a least squares' analysis of values for the linear variation of resistivity vs temperature; for this curve $\mathrm{E}_{\mathrm{f}}=1.27 \times 10^{-2} \mathrm{eV}$. 


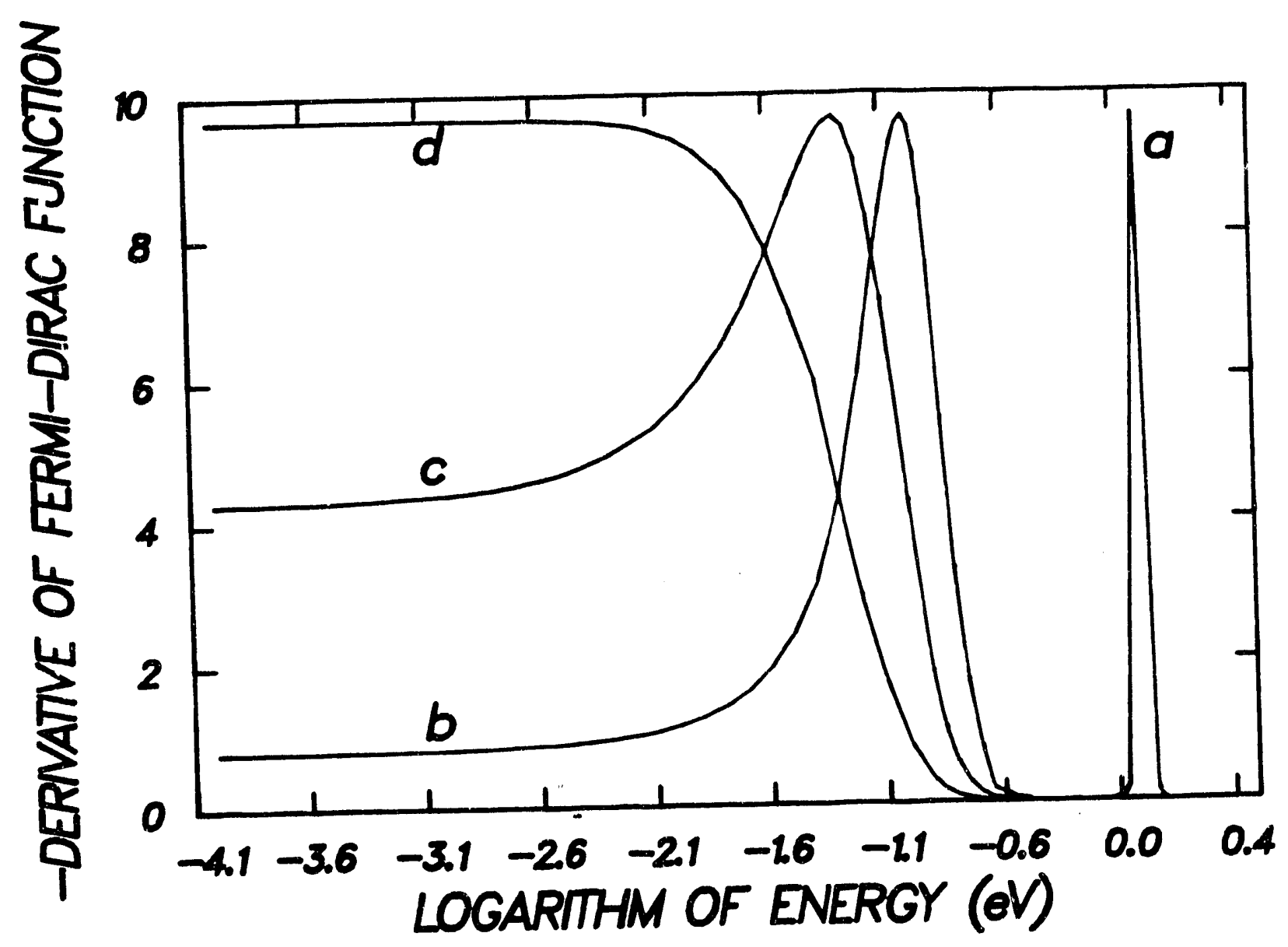




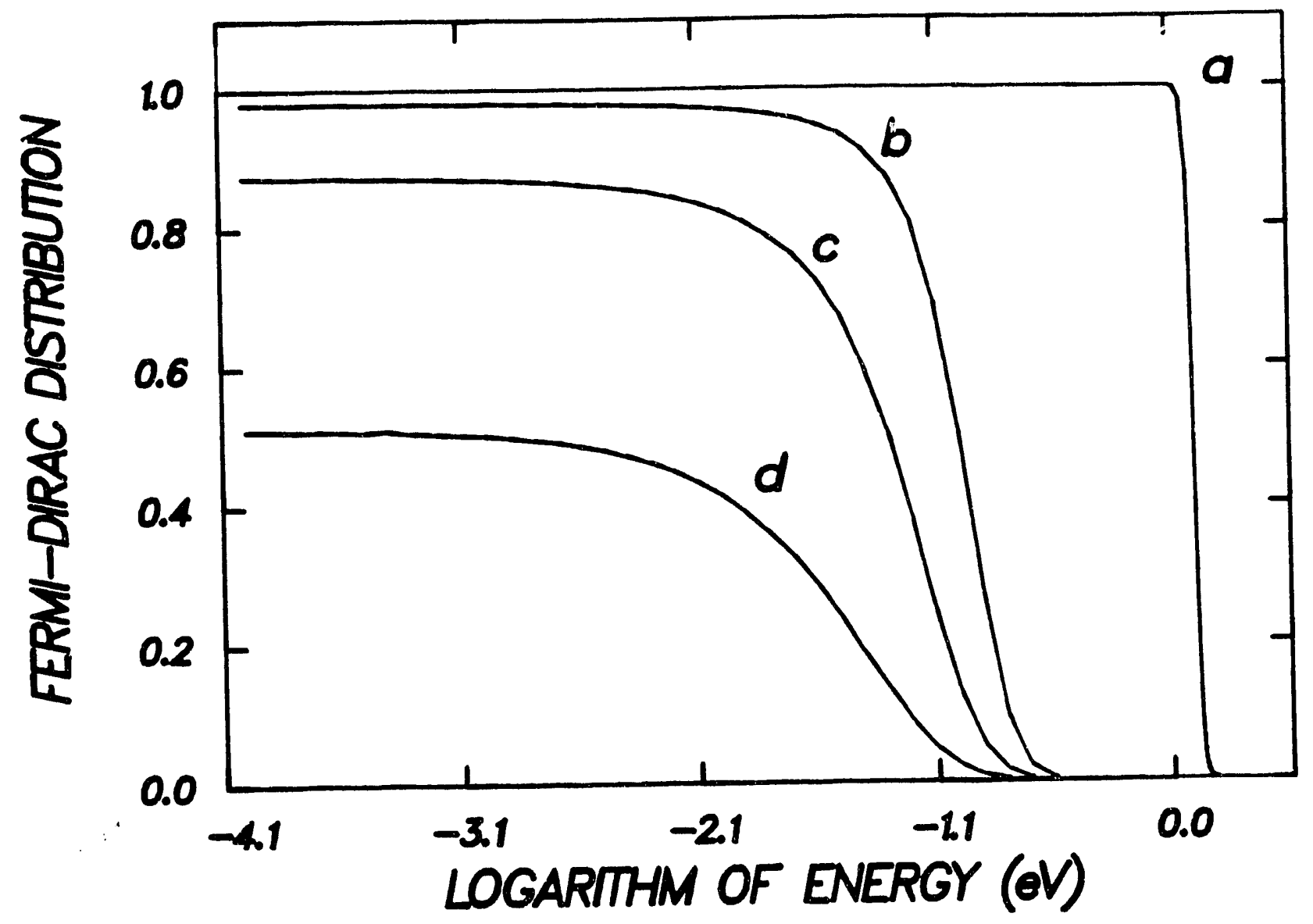




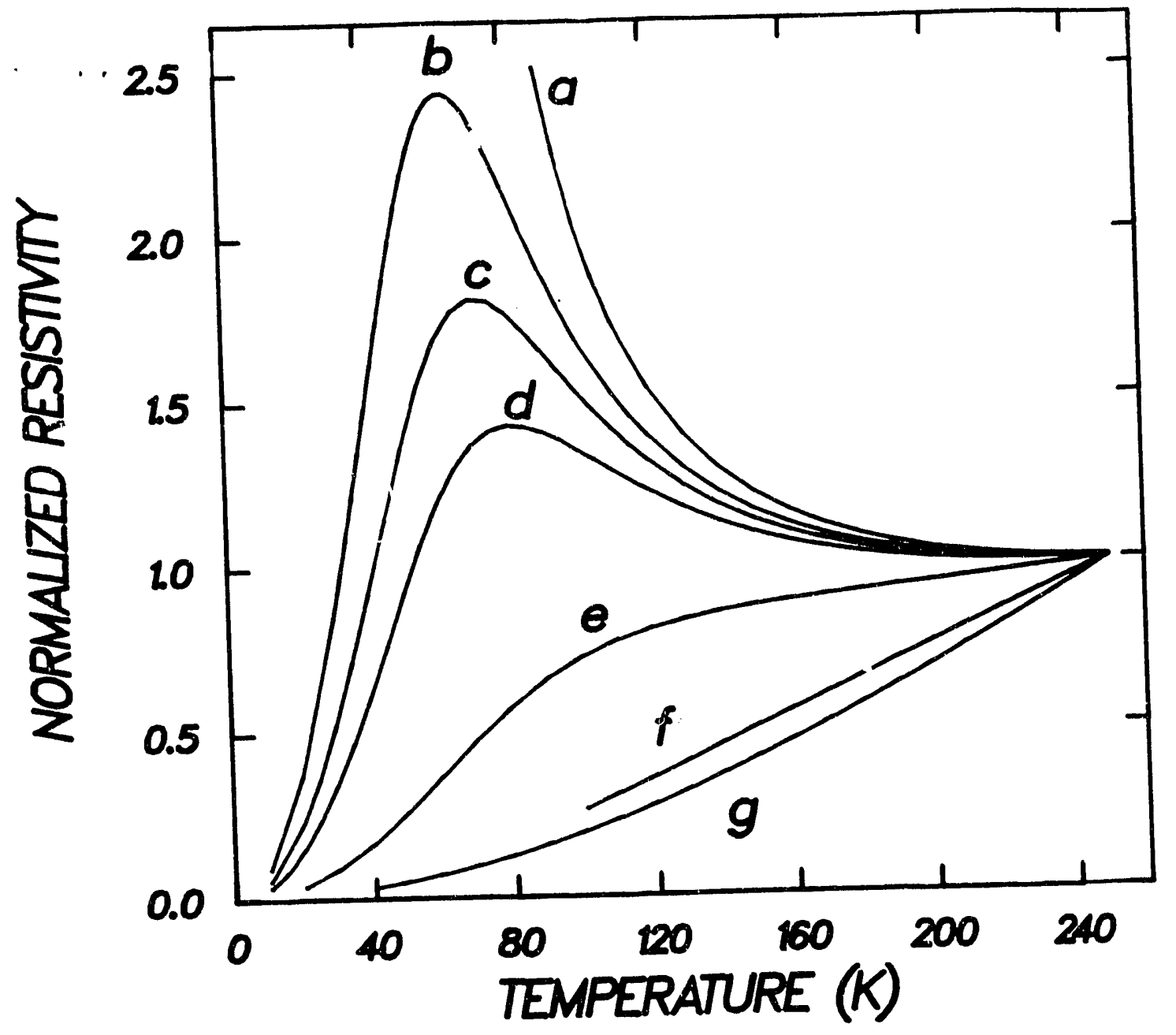



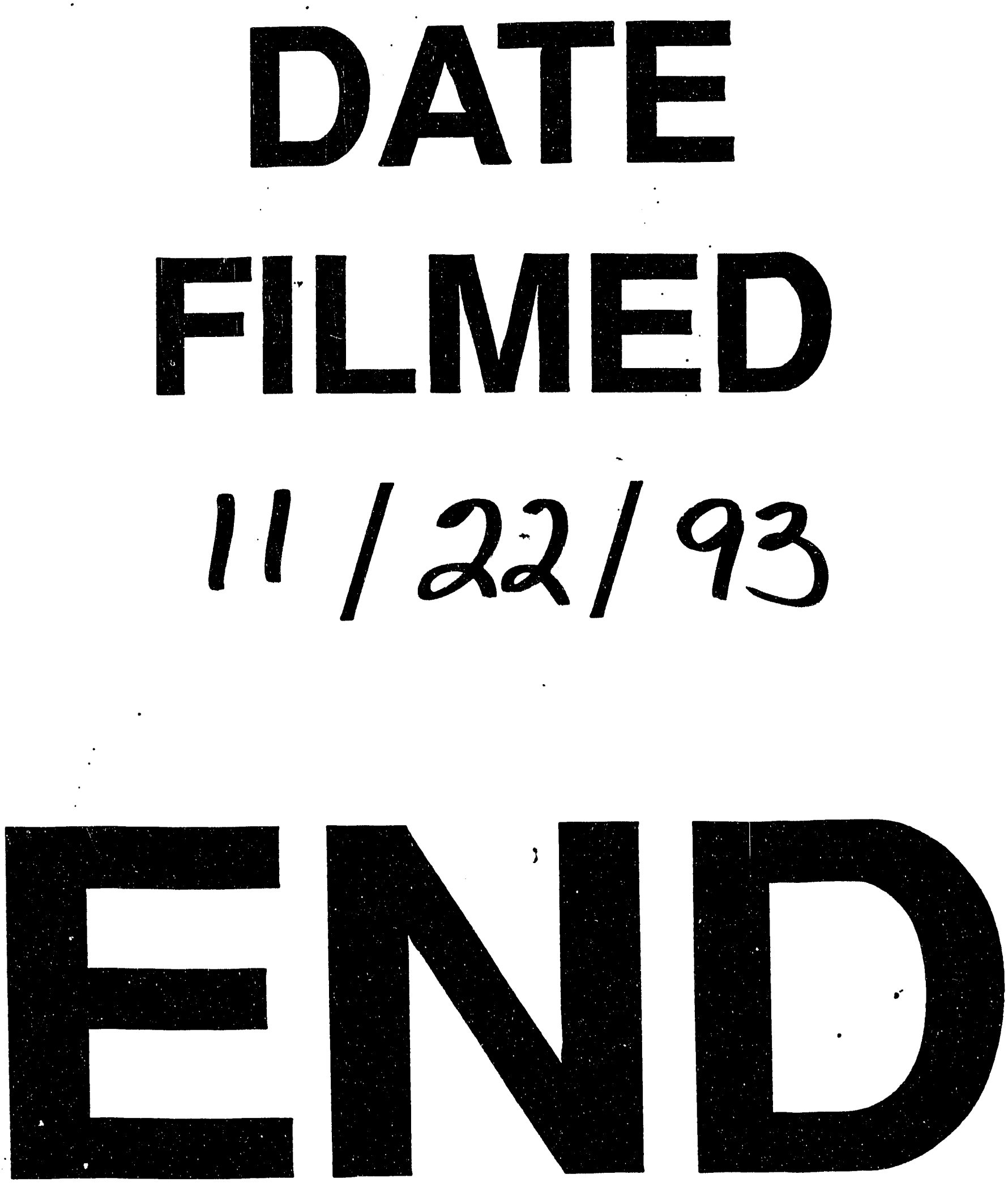
